\title{
Theory of thermostatted inhomogeneous granular fluids: a self-consistent density functional description.
}

\author{
Umberto Marini-Bettolo-Marconi, ${ }^{1}$ Pedro Tarazona, ${ }^{2}$ and Fabio Cecconi ${ }^{3}$ \\ ${ }^{1}$ Dipartimento di Fisica, Via Madonna delle Carceri, 68032 Camerino (MC), Italy \\ ${ }^{2}$ Departamento de Fisica Teórica de la Materia Condensada and Instituto Nicolás Cabrera, \\ Universidad Autonoma de Madrid, E-28049 Madrid, Spain \\ ${ }^{3}$ INFM Center for Statistical Mechanics and Complexity, \\ and Institute for Complex Systems CNR Via dei Taurini 19, 00182 Rome Italy.
}

(Dated: October 26, 2018)

\begin{abstract}
The authors present a study of the non equilibrium statistical properties of a one dimensional hard-rod fluid dissipating energy via inelastic collisions and subject to the action of a Gaussian heat bath, simulating an external driving mechanism. They show that the description of the fluid based on the one-particle phase-space reduced distribution function, in principle necessary because of the presence of velocity dependent collisional dissipation, can be contracted to a simpler description in configurational space. Indeed, by means of a multiple-time scale method the authors derive a self-consistent governing equation for the particle density distribution function. This equation is similar to the dynamic density functional equation employed in the study of colloids, but contains additional terms taking into account the inelastic nature of the fluid. Such terms cannot be derived from a Liapunov generating functional and contribute not only to the relaxational properties, but also to the non equilibrium steady state properties. A validation of the theory against molecular dynamics simulations is presented in a series of cases, and good agreement is found.
\end{abstract}

PACS numbers: 02.50.Ey, 05.20.Dd, 81.05.Rm

\section{INTRODUCTION}

Granular fluids (GFs) represent one of the current paradigms of open non-equilibrium systems and, for this reason, in the last two decades have been the subject of a huge amount of experimental, numerical and theoretical studies $1,2,3,4,5,6.7$. GFs can be conveniently modeled as assemblies of macroscopic particles, experiencing instantaneous binary collisions during which a fraction of the kinetic energy is dissipated, i.e., transferred into internal degrees of freedom. Under the action of a vigorous external driving force, GFs may appear similar to ordinary molecular fluids, but crucial differences remain because inelasticity leads to the appearance of a series of peculiar behaviors, such as clustering, non-Gaussian velocity distribution, and velocity correlations. These phenomena have no counterparts in molecular fluids and render the study of GFs difficult but particularly fascinating.

In spatially uniform systems, relations have been obtained between static average quantities such as density, kinetic temperature and pressure, which may be regarded as the analogue of the equation of state. In addition, a granular hydrodynamics has been developed which, due to the inelasticity of collisions, differs nontrivially from standard hydrodynamics. The majority of these studies focus on large scale properties of the fluid. However, in strongly inhomogeneous systems, the connection between the microscale typical of the particles and the macroscale is still incomplete. Recently, some authors ${ }^{8}$ have proposed phenomenological theories, based on local mass and momentum conservation laws, incorporating nonideal gas effects via an effective free energy functional suitably designed to describe the spontaneous formation of loosely and densely packed regions. This approach sounds very appealing because the free energy density functiona $\underline{1}^{-9}$, besides being a method computationally simple and physically clear, has proven to be a useful tool in the theory of nonuniform fluids with applications to interfacial and freezing phenomena. The basic assumption of all density functional theories is that the thermodynamic potential of a nonuniform system may be approximated knowing the structural and thermodynamic properties of the corresponding uniform system. Two questions are in order before proceeding to generalize the density functional theory (DFT) to granular materials: Does the same method offer any new insight in this new area? How far meaningful concepts for standard molecular fluids, such as free energy and chemical potential, can be extended to systems which are not at thermodynamic equilibrium? The answer to the second question seems to be desolately negative and therefore in order to construct a theory of nonuniform GF, alternative techniques not involving free energy functional derivations have to be developed. A step towards this new direction has been recently made and an equation of evolution for the particle density was obtained in the case of nonequilibrium colloidal systems $\underline{10}$. Interestingly, such an equation is very similar to the equation considered within the dynamic density functional method 11 . It differs from the latter only for the presence of some higher order corrections. The derivation of Ref $\frac{10}{10}$ employs standard tools of kinetic theory and in particular the revised Enskog equation ${ }^{12,13}$. In this paper we generalize such an approach to fluids of inelastic hard core particles subject to a stochastic forcing. 
We consider a one dimensional model of granular fluid which is simple enough as to lend itself to analytic work, but is endowed with a sufficient complexity as to display inhomogeneous behavior $14,15,16,17,18,19,20,21,22,23,24$. One dimensional models may play a useful role since they can be employed to test approximations of more general applicability and allow us to link easily the structural properties to the dynamical behavior. Moreover, at equilibrium the qualitative differences between one dimension and higher dimensions appear in the development of long-range ordering or any kind of phase transitions, but not in the short-range packing structure. In the collision kinetics, the qualitative difference between one dimension and higher dimensions appears in purely inertial and elastic systems through the conservation of the velocity distributions despite the collisions. The role of the bath, and also the inelastic collisions, kills the peculiarity of the one dimensional case. A basic feature of this work is the assumption of a uniform thermostat to describe the external energy supply. The balance between thermostatting and dissipation mechanism gives rise to nonequilibrium steady states which are achieved without fine tuning of the model parameters. Few kinds of thermostats have been employed in the literature, namely, the white noise thermostat $\stackrel{25}{2}$, the Gaussian thermostat 26 and the Langevin thermostat $27,28,29$ which includes both the white noise term and the friction force proportional to the velocity of the particles ${ }^{30}$. The present study is based on the Langevin thermostat because it can be easily realized in numerical experiments and lends itself to a great deal of analytical work.

The paper is organized as follows. In Sec. II] we introduce the equations describing the dynamics of the stochastically driven inelastic hard-rod fluid model. We start from the stochastic equations for the trajectories of each particle and discuss how these can be reduced under some suitable approximations to the Fokker-Planck-Boltzmann equation for the single-particle phase-space distribution. Next, in order to make analytical progress, we separate the velocity and the spatial dependence of the distribution function and obtain an infinite hierarchy of coupled integro-differential equations. In Sec. [II] we analyze the steady state uniform properties of the system. In Sec. [V] we introduce the evolution equation for the density of the system, which is obtained in Appendix A by applying the multiple time scale method, and in Sec. $\mathrm{V}$ we perform a series of numerical tests of our theory using a few selected examples. The tests compare the two levels of description: the results obtained studying the single particle trajectories with those obtained from the density equation. Finally, conclusions are drawn in Sec. [VI]

\section{MODEL}

Let us consider a one dimensional fluid consisting of $N$ identical inelastic hard rods of mass $m$, length $\sigma$, coefficient of restitution $\alpha$, positions $x_{i}$, and velocities $v_{i}$, with $i=1, \ldots, N$. For the sake of generality we also consider an arbitrary external force, $f_{\text {ext }}(x)$. When the separation of particles $i$ and $i+1$ is $\sigma$ a binary inelastic collision occurs. The collision conserves the total momentum and is described by the linear transformation:

$$
\begin{aligned}
& v_{1}^{\prime}=v_{1}+\frac{1+\alpha}{2 \alpha}\left(v_{2}-v_{1}\right) \\
& v_{2}^{\prime}=v_{2}-\frac{1+\alpha}{2 \alpha}\left(v_{2}-v_{1}\right)
\end{aligned}
$$

connecting the precollisional velocities (primed symbols) and the postcollisional velocities (unprimed symbols). Since in a single collision the amount of total kinetic energy,

$$
\Delta E=-\frac{m}{4}\left(1-\alpha^{2}\right)\left(v_{1}^{\prime}-v_{2}^{\prime}\right)^{2},
$$

is dissipated, without energy injection the particles would come to rest. On the contrary, a steady state regime can be reached if the energy loss through collisions is balanced by an energy injection that we assume to be realized by the combination of a friction force $-m \gamma v_{i}$ and a stochastic force $\xi_{i}(t)$. These two forces represent the Langevin thermostat. The complete dynamics can be represented by the following $2 \mathrm{~N}$ coupled stochastic differential equations:

$$
\begin{aligned}
\frac{d x_{i}}{d t} & =v_{i} \\
m \frac{d v_{i}}{d t} & =-m \gamma v_{i}+f_{\text {ext }}\left(x_{i}\right)+f_{i}^{c o l l}+\xi_{i}(t)
\end{aligned}
$$

where $f_{i}^{\text {coll }}$ indicates symbolically the resultant of the impulsive forces acting on particle $i$ in possible hard core collisions against other particles. The stochastic force $\xi_{i}(t)$ has zero average $\left\langle\xi_{i}(t)\right\rangle$ and white noise correlation

$$
\left\langle\xi_{i}(t) \xi_{j}\left(t^{\prime}\right)\right\rangle=2 \gamma m T_{0} \delta_{i j} \delta\left(t-t^{\prime}\right),
$$

The amplitude $T_{0}$ is the "heat-bath temperature" and $\langle\cdot\rangle$ indicates the average over a statistical ensemble of realizations of the noise. A statistical description of the system in terms of the one-particle phase space distribution $f^{(1)}(x, v, t)$, 
giving the number of particles in the volume element $(x, x+d x, v, v+d v)$, can be worked out by taking the average over all realizations of the stochastic noise (see. Ref 10 ). Moreover, the distribution $f^{(1)}(x, v, t)$ evolves according to the governing equation

$$
\frac{\partial}{\partial t} f^{(1)}(x, v, t)+\left[v \frac{\partial}{\partial x}+\frac{f_{e x t}(x)}{m} \frac{\partial}{\partial v}\right] f^{(1)}(x, v, t)=\gamma\left[\frac{\partial}{\partial v} v+\frac{T_{0}}{m} \frac{\partial^{2}}{\partial v^{2}}\right] f^{(1)}(x, v, t)+k(x, v, t) .
$$

In the left hand side, the term between the square brackets describes the free streaming of the particles subject to the external force $f_{e}(x)$, the first term in the right hand side is the one-particle Fokker-Planck collision term representing the interaction with the heat bath, while $k(x, v, t)$ describes the collisions among the particles. We treat these interactions within the revised Enskog theory (RET), developed by Ernst and van Beijeren $\stackrel{12}{ }$. The RET for elastic collisions is accurate over the entire fluid range and describes the crystal phase too. It has been generalized to the inelastic regime and used to derive transport coefficients ${ }^{31.32}$. We write the RET collision operator as:

$$
\begin{aligned}
& k(x, v, t)=\sum_{s= \pm 1} \int d v_{2} \Theta\left(s v_{12}\right)\left(s v_{12}\right) \\
& \times\left[\frac{1}{\alpha^{2}} g_{2}(x, x-s \sigma \mid \rho) f^{(1)}\left(x, v_{1}^{\prime}, t\right) f^{(1)}\left(x-s \sigma, v_{2}^{\prime}, t\right)-g_{2}(x, x+s \sigma \mid \rho) f^{(1)}\left(x, v_{1}, t\right) f^{(1)}\left(x+s \sigma, v_{2}, t\right)\right]
\end{aligned}
$$

Notice that at variance with the elastic case, a quadratic factor $\alpha^{-2}$ in the gain term, specific to granular gases, appears. One power is the consequence of the Jacobian $d v_{1}^{\prime} d v_{2}^{\prime}=\frac{1}{\alpha} d v_{1} d v_{2}$ and the second power stems from the reflection law $v_{12}=-\alpha v_{12}^{\prime}$. The sum over $s= \pm 1$ is the analogue in $d=1$ of the integration over the d-dimensional surface of the hyper-sphere with radius $\sigma$. The RET embodies spatial correlations through the hard-rod pair correlation function, $g_{2}(x, x \pm \sigma ; n)$ evaluated at contact. As a simplifying approximation, to obtain a theory at the level of the one-particle distribution, we take $g_{2}(x, x \pm \sigma \mid \rho)$ to be given by its equilibrium value ${ }^{33}$ evaluated when the local density is $\rho(x, t)$ :

$$
g_{2}(x \pm \sigma \mid \rho)=\frac{1}{1-\eta\left(x \pm \frac{\sigma}{2}\right)} .
$$

The time and density dependence occurs entirely via the local packing fraction $\eta(x, t)=\int_{x-\sigma / 2}^{x+\sigma / 2} d x^{\prime} \rho\left(x^{\prime}, t\right)$. Therefore, the collision operator Eq. (8) is approximated by an explicit non-local functional of the one-particle density distribution, with the terms $f^{(1)}\left(x, v_{1}, t\right) f^{(1)}\left(x \pm \sigma, v_{2}, t\right)$, set by the collision distance, and the nonlocal density dependence through $\eta(x \pm \sigma / 2)$, to include the particle correlations.

In the following we shall employ the non dimensional set of variables which are obtained by measuring the velocities in units of the thermal velocity $v_{T}=\sqrt{k_{B} T_{0} / m}$ and lengths in unit of $\sigma$, i.e. $V \equiv v / v_{T}$ and $X \equiv x / \sigma$. The remaining variables can be non-dimensionalized according to the transformations $\left.\tau \equiv t v_{T} / \operatorname{sigma}\right), \Gamma=\gamma \sigma / v_{T} . \quad F(X) \equiv$ $\sigma f_{e x t}(x) / m v_{T}^{2}$. Finally, the distribution function and the collision term are rescaled according to the transformations: $P(X, V, \tau) \equiv \sigma v_{T} f^{(1)}(x, v, t)$ and $K(X, V, \tau) \equiv \sigma^{2} k(x, v, t)$.

Equation (6) can be cast in the following non dimensional form:

$$
\frac{1}{\Gamma} \frac{\partial P(X, V, \tau)}{\partial \tau}=L_{F P} P(X, V, \tau)-\frac{1}{\Gamma} V \frac{\partial}{\partial X} P(X, V, \tau)-\frac{1}{\Gamma} F(X, \tau) \frac{\partial}{\partial V} P(X, V, \tau)+\frac{1}{\Gamma} K(X, V, \tau)
$$

where we have introduced Fokker-Planck operator, $L_{F P}$ by the equation:

$$
L_{F P} P(X, V, \tau)=\frac{\partial}{\partial V}\left[\frac{\partial}{\partial V}+V\right] P(X, V, \tau)
$$

The eigenfunctions of $L_{F P}$ read explicitly

$$
H_{\nu}(V) \equiv \frac{1}{\sqrt{2 \pi}}(-1)^{\nu} \frac{\partial^{\nu}}{\partial V^{\nu}} \exp \left(-\frac{1}{2} V^{2}\right)
$$

and correspond to discrete eigenvalues $\nu=0,-1,-2, \ldots$. We separate the velocity from the spatial dependence by expanding, over the basis set $H_{\nu}(V)$, both the phase-space distribution

$$
P(X, V, \tau)=\sum_{\nu} \Phi_{\nu}(X, \tau) H_{\nu}(V)
$$


and the collision term

$$
K(X, V, \tau)=\sum_{\nu} C_{\nu}(X, \tau) H_{\nu}(V)
$$

As shown in Appendix A, the coefficients $C_{\nu}(X, \tau)$ can be expressed as nonlocal products of the moments $\Phi_{\nu}(X, \tau)$ with coefficients which are nonlocal functionals of the density distribution. Substituting Eqs.(12) and (13) in Eq. (9) and using the orthogonality of the basis set $H_{\nu}(V)$, we obtain a system of coupled equations: for the moments $\Phi_{\nu}(X, \tau)$ which can be written in compact form as:

$$
\left[\frac{\partial \Phi_{\nu}(X, \tau)}{\partial \tau}+\Gamma \nu \Phi_{\nu}(X, \tau)-C_{\nu}(X, \tau)\right]+(\nu+1) \frac{\partial \Phi_{\nu+1}(X, \tau)}{\partial X}+\left[\frac{\partial}{\partial X}-F(X)\right] \Phi_{\nu-1}(X, \tau)=0
$$

with $\Phi_{-1}=0$.

We identify the moment $\Phi_{0}(X, \tau)=\int d V P(X, V, \tau)$ with the number density, $\Phi_{1}(X, \tau)=\int d V V P(X, V, \tau)$ with the momentum density and $\Phi_{2}(X, \tau)+\Phi_{0}(X, \tau) / 2=1 / 2 \int d V V^{2} P(X, V, \tau)$ with the kinetic energy density. For $\nu=0,1,2$, Eq. (14) encodes the balance equations for these moments, i.e. the hydrodynamic equations characterizing a viscous onedimensional fluid ${ }^{34}$. Finally, we introduce a local kinetic temperature, often called granular temperature, via the definition:

$$
T(X, \tau)=\left(\left\langle V^{2}\right\rangle-\langle V\rangle^{2}\right)=1+2 \frac{\Phi_{2}(X, \tau)}{\Phi_{0}(X, \tau)}-\left[\frac{\Phi_{1}(X, \tau)}{\Phi_{0}(X, \tau)}\right]^{2}
$$

\section{HOMOGENEOUS STEADY STATE PROPERTIES}

Before embarking upon the task of solving the evolution equations, we illustrate the peculiarity of the inelastic system by choosing the simplest case, namely, a time-independent spatially uniform system with $F(X)=0$. We consider the global velocity distribution function $\Psi(V)$, and show that in the steady state it does not relax to the Maxwellian, as it would occur in the case of a molecular fluid. To this purpose let $\Phi_{i}=\tilde{\Phi}_{i}$, where $\tilde{\Phi}_{i}$ are some constants. The value of the amplitude $\tilde{\Phi}_{2}$, through Eq. (14), can be expressed in terms of the uniform density $\tilde{\Phi}_{0}$ :

$$
2 \Gamma \tilde{\Phi}_{2}=C_{2}=-\frac{\left(1-\alpha^{2}\right)}{\sqrt{\pi}} g_{2}\left[\tilde{\Phi}_{0}^{2}+3 \tilde{\Phi}_{0} \tilde{\Phi}_{2}+\frac{3}{4} \tilde{\Phi}_{2}^{2}\right]
$$

where the second equality follows from the definition of $C_{2}$ and the expressions of appendix A. To first order in the inelasticity parameter $\left(1-\alpha^{2}\right)$ we obtain: $\tilde{\Phi}_{2}=-\epsilon \tilde{\Phi}_{0} / 2$, where $\epsilon=\left[\left(1-\alpha^{2}\right) /(\sqrt{\pi} \Gamma)\right] g_{2} \tilde{\Phi}_{0}$. The procedure can be carried on for values of $\nu$ larger than 2 with the following result:

$$
\begin{gathered}
\tilde{\Phi}_{4}=\frac{1}{4 \Gamma} C_{4} \simeq \frac{\left(1-2 \alpha^{2}\right)}{96} \epsilon \tilde{\Phi}_{0} \\
\tilde{\Phi}_{6}=\frac{1}{6 \Gamma} C_{6} \simeq-\frac{\left(3-12 \alpha^{2}+8 \alpha^{4}\right)}{5760} \epsilon \tilde{\Phi}_{0} \\
\tilde{\Phi}_{8}=\frac{1}{8 \Gamma} C_{8} \simeq-\frac{\left(15-20 \alpha^{2}+50 \alpha^{4}-16 \alpha^{6}\right)}{215040} \epsilon \tilde{\Phi}_{0}
\end{gathered}
$$

Hence, $\Psi(V)$ can be written as

$$
\begin{aligned}
\Psi(V)= & \frac{e^{-V^{2} / 2}}{\sqrt{2 \pi}}\left[\tilde{\Phi}_{0}+\left(V^{2}-1\right) \tilde{\Phi}_{2}+\left(V^{4}-6 V^{2}+3\right) \tilde{\Phi}_{4}+\right. \\
& \left.\left(V^{6}-15 V^{4}+45 V^{2}-15\right) \tilde{\Phi}_{6}+\left(V^{8}-28 V^{6}+210 V^{4}-420 V^{2}+105\right) \tilde{\Phi}_{8}\right] .
\end{aligned}
$$

Two remarks are in order: if we retain only the two leading terms in the expansion, the distribution function can be approximately rewritten as a Maxwellian,

$$
\Psi(V)=\tilde{\Phi}_{0}\left[1-\left(V^{2}-1\right) \frac{\epsilon}{2}\right] \frac{e^{-V^{2} / 2}}{\sqrt{2 \pi}} \simeq \frac{\exp \left(-\frac{V^{2}}{2(1-\epsilon)}\right)}{\sqrt{2 \pi(1-\epsilon)}} \tilde{\Phi}_{0}
$$


and we interpret $\epsilon$ as the reduced temperature shift induced by the inelastic dissipation. Secondly, the expansion Eq. (21) can be compared with an exact solution of Eq. (9), valid when $F(X)=0$ and in the limit $(1-\alpha) \rightarrow 0^{35}$, obtained by Benedetto et al.. These authors showed that a spatially uniform solution, $\Psi_{p}(V)$, of Eq. (9) is given implicitly by the following nonlinear integral equation:

$$
\Psi_{p}(V)=\frac{e^{-V^{2} / 2}}{\sqrt{2 \pi} Z} \exp \left\{-\frac{(1-\alpha) g_{2}}{6 \Gamma}\left[\int_{0}^{\infty} d u u^{3} \Psi_{p}(u+V)-\int_{-\infty}^{0} d u u^{3} \Psi_{p}(u+V)\right]\right\}
$$

where $Z$ is the constant which ensures the correct normalization of the probability distribution function (PDF). Interestingly, such a distribution has high-velocity tails which decay as $\exp \left(-c|V|^{3}\right)$, whereas the central region of the distribution is approximately a Maxwellian. Clearly, the high-velocity tails cannot be well reproduced by the present expansion, which is applicable when $\Gamma>>1$, but the kurtosis associated with Eq. (21) compares reasonably with the kurtosis computed from the distribution $P_{p}(V)$, as shown in Fig. 1,

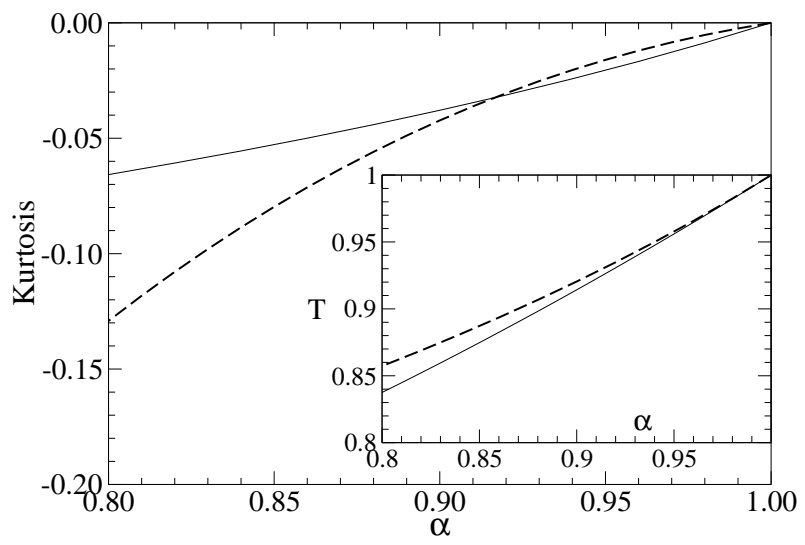

FIG. 1: Kurtosis of the velocity distribution as a function of $\alpha$ for $\rho_{0}=0.8$ and $\Gamma=5$. The dashed line represents the data of the present theory, whilst the continuous line refers to the results from formula (22) by Pulvirenti and coworkers. In the inset we display the corresponding kinetic temperatures.

\section{EVOLUTION EQUATION}

We shall consider, in the following, the nearly overdamped regime $\Gamma>1$ 36,37,38,39,40,41,42. Since only the particle number is conserved, one expects that after a transient of duration of the order of $\gamma^{-1}$, the momentum and the energy current become slaved by the density field. This remark allows us to simplify the task posed by the open hierarchy of Eqs. (14). In Ref ${ }^{10}$, we showed that, for a system undergoing perfectly elastic collisions, the problem can be treated conveniently by employing a multiple-time scale technique. As a result we found a reaction-diffusion self-consistent equation involving only the amplitude $\Phi_{0}(X, \tau)$. The evolution of all remaining partial amplitudes $\Phi_{\nu}(X, \tau),(\nu>1)$ could be deduced from the knowledge of $\Phi_{0}(X, \tau)$. Physically, the reason for such a complexity reduction can be attributed to the fact that the marginal velocity probability distribution attains its local equilibrium rapidly, in a time span of the order of $\gamma^{-1}$, during which the one-particle density changes slowly. Indeed, the positional degrees of freedom reach an equilibrium distribution on a much slower time scale than the velocities.

Since the method of solution follows closely the derivation of Ref 10 , we report the details of the present case in Appendix B and proceed to illustrate the resulting equation of evolution. We only recall that the method is based on a systematic expansion in powers of $\Gamma^{-1}$ which takes into account the fact that in Eq. (9) the time derivative is multiplied by the small parameter $\Gamma^{-1}$. For such a reason a multiple time-scale method has to be applied. We also need to introduce the following expansions of the moments and of the collision integrals in inverse powers of $\Gamma$ :

$$
\Phi_{\nu}(X, \tau)=\sum_{n} \frac{1}{\Gamma^{n}} \phi_{n \nu}(X, \tau)
$$

and

$$
C_{\nu}(X, \tau)=\sum_{n} \frac{1}{\Gamma^{n}} c_{n \nu}(X, \tau)
$$


The key result of the analysis contained in Appendix B is the following equation for the density amplitude $\phi_{00}$ :

$$
\frac{\partial \phi_{00}}{\partial \tau}(X, \tau)=\frac{1}{\Gamma} \partial_{X}\left\{\left[\partial_{X}-F(X)\right] \phi_{00}(X, \tau)-c_{01}(X, \tau)-\frac{1}{\Gamma} c_{11}(X, \tau)+\frac{1}{\Gamma} \partial_{X} c_{02}(X, \tau)\right\} .
$$

Equation (25) is the fundamental equation of this work and constitutes a closed expression, once the collisional terms $c_{s \nu}$ and the amplitudes $\phi_{s \nu}$ are specified in terms of the scaled density $\phi_{00}(X, \tau)$. As we will show below, the density field $\phi_{00}(X, \tau)$ fully characterizes the state of the system and slaves the remaining hydrodynamic fields. Indeed, the amplitudes of the $H_{1}(V)$ and $H_{2}(V)$ components are completely determined from the knowledge of $\phi_{00}(X, \tau)$. Such a complexity reduction occurs because the density is the only conserved field in our thermostatted model.

Using Eq. (B4) we obtain at order $\Gamma^{-1}$, the following:

$$
c_{01}(X, \tau)=-\frac{(1+\alpha)}{2} \phi_{00}(X, \tau)\left[g_{2}(X, X+1) \phi_{00}(X+1, \tau)-g_{2}(X, X-1) \phi_{00}(X-1, \tau)\right]
$$

and the following at order $\Gamma^{-2}$ :

$$
c_{02}(X, \tau)=-\frac{\left(1-\alpha^{2}\right)}{2 \sqrt{\pi}} \phi_{00}(X, \tau)\left[g_{2}(X, X+1) \phi_{00}(X+1, \tau)+g_{2}(X, X-1) \phi_{00}(X-1, \tau)\right]
$$

and

$$
\begin{aligned}
c_{11}(X, \tau)= & \\
& \frac{(1+\alpha)}{\sqrt{\pi}} \phi_{00}(X, \tau)\left[g_{2}(X, X+1) \phi_{11}(X+1, \tau)+g_{2}(X, X-1) \phi_{11}(X-1, \tau)\right] \\
- & \frac{(1+\alpha)}{\sqrt{\pi}} \phi_{11}(X, \tau)\left[g_{2}(X, X+1) \phi_{00}(X+1, \tau)+g_{2}(X, X-1) \phi_{00}(X-1, \tau)\right] \\
& -\frac{(1+\alpha)}{2} \phi_{00}(X, \tau)\left[g_{2}(X, X+1) \phi_{12}(X+1, \tau)-g_{2}(X, X-1) \phi_{12}(X-1, \tau)\right] \\
& -\frac{(1+\alpha)}{2} \phi_{12}(X, \tau)\left[g_{2}(X, X+1) \phi_{00}(X+1, \tau)-g_{2}(X, X-1) \phi_{00}(X-1, \tau)\right]
\end{aligned}
$$

where

$$
\phi_{11}(X, \tau)=-\left[\partial_{X}-F(X)\right] \phi_{00}(X, \tau)+c_{01}(X, \tau)
$$

and

$$
\phi_{12}(X, \tau)=\frac{1}{2} c_{02}(X, \tau) .
$$

It is now clear that the quantities $c_{s \nu}(X, \tau)$, which depend locally on time but nonlocally on space, play the role of effective fields because they encode the influence of the remaining particles on the particle located at $X$. They are also functionals of the scaled density $\phi_{00}(X, \tau)$, so that Eq. (25) is self-consistent and can be solved numerically by iteration. Relation (25) is a continuity equation for the particle density, whose current can be written as $\Phi_{1}(X, \tau)=$ $\phi_{11}(X, \tau) / \Gamma+\phi_{21}(X, \tau) / \Gamma^{2}$.

Interestingly, for $\alpha=1$, Eq. ((29) $)$ can be recast to

$$
\phi_{11}(X, \tau)=-\phi_{00}(X, \tau) \partial_{X}\left[\frac{\delta F^{r o d}\left[\phi_{00}\right]}{\delta \phi_{00}(X, \tau)}+V_{e x t}(X)\right],
$$

where $F^{\text {rod }}\left[\phi_{00}\right]$ is the hard rod density functional of the instantaneous density $\phi_{00}(X, \tau)$.

The term $c_{02}$ vanishes in the limit $\alpha \rightarrow 0$ and describes a tendency of the particles to form denser aggregates due to their inelasticity.

Also notice that at the zero order in $\Gamma^{-1}$, Eq. (25) may be rewritten as a dynamic density functional (DDF) equation $\underline{10}$, since the only change with respect to the $\alpha=1$ case $\frac{11}{1}$ is the presence of a prefactor $(1+\alpha) / 2$ in the $c_{01}$ term in Eq. (26). Hence, if we define an inelastic free energy density functional as $F_{\alpha}[\rho]=F_{\text {ideal }}[\rho]+(1+\alpha) / 2 F_{\text {excess }}[\rho]$ scaling the exact hard-rods excess of the equilibrium case, we would cast Eq. (25) into a DDF equation for arbitrary values of $\alpha$. A particular result would be that, always at the leading order in $\Gamma^{-1}$, the equilibrium density profiles should be given by the minimum of such inelastic free energy density functional. The results in Figs. 5 and 7 are qualitatively consistent with that effect since the reduction of the excess free energy reduces the oscillations. As we shall see below the equation of state for the uniform fluid (Eq. (40)) predicts a lowering of the pressure with respect to the elastic case also consistent with such a scaling of the nonideal part of the free energy.

Hereafter, we briefly derive some useful relations between the density profile, the temperature and the pressure in the non uniform steady state where the current $\Phi_{1}$ vanishes. 


\section{A. Steady state temperature profile}

In the limit $\tau \rightarrow \infty$ we determine the granular temperature profile, using the previous results and Eq. (15):

$$
T(X)=1+\frac{2}{\Gamma} \frac{\phi_{12}(X)}{\phi_{00}(X)}=1-\frac{\left(1-\alpha^{2}\right)}{2 \Gamma \sqrt{\pi}}\left[g_{2}(X, X+1) \phi_{00}(X+1, \tau)+g_{2}(X, X-1) \phi_{00}(X-1)\right]
$$

where we suppressed the time argument and the functions of the single spatial argument have to be understood as their asymptotic limiting values when $\tau \rightarrow \infty$. The constant 1 in the r.h.s. represents (in our reduced units) the heat-bath temperature, whereas the second term is the shift in the local temperature induced by collisions. In fact, it amounts to the product of three factors: the kinetic energy dissipated, the collision rate $\omega_{E}$ (Enskog collision frequency ${ }^{43}$ ) and the typical time $\gamma^{-1}$ of the heat-bath. The average Enskog frequency at each side of the particle located at $X$ is:

$$
\frac{\omega_{E}(X \pm 1)}{\gamma}=\frac{2}{\Gamma \sqrt{\pi}} g_{2}(X, X \pm 1) \phi_{00}(X \pm 1)
$$

and in the case of a uniform system it reduces to the bulk Enskog frequency $\omega_{E}=2 v_{T} \rho \sigma g_{2} / \sqrt{\pi}$. where $v_{T}$ is the thermal velocity of the gas.

\section{B. Steady state pressure profile.}

We now turn our attention to the pressure profile $\Pi(X, \tau)$, which can be separated into a kinetic and a collisional contribution

$$
\Pi(X)=\Pi_{k i n}(X)+\Pi_{\text {coll }}(X) .
$$

The total pressure $\Pi(X, \tau)$ is implicitly determined from the momentum balance equation, obtained by considering Eq. (14) with $\nu=1$ in the $\tau \rightarrow \infty$ limit

$$
F(X) \Phi_{0}(X)-\frac{\partial\left[\Pi_{k i n}(X)+\Pi_{\text {coll }}(X)\right]}{\partial X}=0 .
$$

We identify the first term as

$$
\Pi_{k i n}(X)=\int d V V^{2} P(X, V)=\Phi_{0}(X)+2 \Phi_{2}(X)
$$

or using the results of the $\Gamma$ expansion, we rewrite

$$
\Pi_{k i n}(X)=\phi_{00}(X)+\frac{2}{\Gamma} \phi_{12}(X),=T(X) \phi_{00}(X)
$$

where we used Eq. (31) to obtain the last equality. In Ref. 10 we showed that the spatial derivative of the collisional pressure is related to the collision integral via the relation

$$
\partial_{X} \Pi_{\text {coll }}(X)=-C_{1}(X)=-c_{01}(X)-\frac{1}{\Gamma} c_{11}(X) .
$$

By manipulating expressions (26) and (28) (see Ref. 10 for details) we formally integrate Eq. (37) with the following result:

$$
\begin{aligned}
& \Pi_{\text {coll }}(X)=\frac{(1+\alpha)}{2} \int_{0}^{1} d z g_{2}(X-(1-z), X+z) \times \\
& \left\{\phi_{00}(X-(1-z)) \phi_{00}(X+z)\right. \\
& +\frac{1}{\Gamma}\left[\phi_{00}(X-(1-z)) \phi_{12}(X+z)+\phi_{12}(X-(1-z)) \phi_{00}(X+z)\right] \\
& \left.-\frac{2}{\Gamma \sqrt{\pi}}\left[\phi_{00}(X-(1-z)) \phi_{11}(X+z)-\phi_{11}(X-(1-z)) \phi_{00}(X+z)\right]\right\} .
\end{aligned}
$$


In the case of constant density, the z-integration can be trivially performed, and we obtain

$$
\Pi_{\text {coll }}=\frac{(1+\alpha)}{2} \frac{\phi_{00}^{2}}{1-\phi_{00}}\left(1+\frac{2}{\Gamma} \frac{\phi_{12}}{\phi_{00}}\right) .
$$

Finally, by using Eq. (36) we cast the equation of state in the uniform non equilibrium steady state in the form:

$$
\Pi=T \phi_{00}\left[1+\frac{(1+\alpha)}{2} \frac{\phi_{00}}{1-\phi_{00}}\right] .
$$

Expressing the temperature as a function of the density (from Eq. (31))

$$
T=1-\frac{\left(1-\alpha^{2}\right)}{\Gamma \sqrt{\pi}} g_{2} \phi_{00}
$$

we see that Eq. (40) describes the lowering of the pressure due to the collisional reduction of the temperature and becomes the familiar hard-rod pressure equation for $\alpha=1$.

\section{APPLICATIONS}

\section{A. Temporal decay of a small density modulation}

We begin by considering the decay of an infinitesimal sinusoidal perturbation of wave-vector $K$ with respect to a uniform density profile and how the relaxation time varies as a function of $K$. We assume that the sinusoidal density perturbation is small with respect to some uniform background density, $\Phi_{0}$ and write:

$$
\phi_{00}(X, \tau)=\Phi_{0}+\hat{\rho}_{K}(\tau) \sin (K X)
$$

After some simple algebra we arrive at the following equation of evolution for the modulation:

$$
\begin{aligned}
\frac{\partial \rho_{K}(\tau)}{\partial \tau}= & -R(K) \rho_{K}(\tau) \\
= & -\frac{K^{2}}{\Gamma} \rho_{K}(\tau)\left\{\left[1+\frac{(1+\alpha)}{2}\left(2 p_{0} \sigma \frac{\sin (K)}{K}+\frac{4\left(p_{0} \sigma\right)^{2}}{K^{2}} \sin ^{2}(K / 2)\right)\right]\right. \\
& {\left[1-2 \frac{(1+\alpha)}{\Gamma \sqrt{\pi}} p_{0} \sigma(1-\cos (K))\right] } \\
- & \frac{\left(1-\alpha^{2}\right)}{\Gamma \sqrt{\pi}} \frac{(1+\alpha)}{2} p_{0} \sigma \frac{1}{2}\left[2 p_{0} \sigma \frac{\sin (K)}{K}+\frac{4\left(p_{0} \sigma\right)^{2}}{K^{2}} \sin ^{2}(K / 2)\right] \\
- & \left.\frac{\left(1-\alpha^{2}\right)}{\Gamma \sqrt{\pi}}\left[p_{0} \sigma(1+\cos (K))+\left(p_{0} \sigma\right)^{2} \frac{\sin (K)}{K}\right]\left[1+\frac{(1+\alpha)}{2} p_{0} \sigma \frac{\sin (K)}{K}\right]\right\}
\end{aligned}
$$

with $p_{0}=\Phi_{0} /\left(1-\Phi_{0}\right)$ being the bulk hard rod reduced pressure. Taking the long wavelength limit $K \rightarrow 0$, we obtain the following expression for the diffusion coefficient:

$$
D=\lim _{K \rightarrow 0} \frac{R(K)}{K^{2}}=\frac{1}{\Gamma}\left\{1+(1+\alpha)\left(p_{0} \sigma+p_{0}^{2} \sigma^{2} / 2\right)-\frac{\left(1-\alpha^{2}\right)}{\Gamma \sqrt{\pi}}\left(2+3 \frac{1+\alpha}{2} p_{0} \sigma\right)\left(p_{0}+p_{0}^{2} \sigma / 2\right)\right\}
$$

As shown in Fig. 2] the relaxation time of the inelastic fluid, for small values of $K$, is longer than the corresponding quantity in the elastic fluid. This occurs because, being the granular temperature of the former lower, the diffusion is weaker. In addition, while the excluded volume favors diffusion with respect to the non interacting case, the inelasticity operates in the opposite direction. For $K \rightarrow 0$ the temperature field $T(X, \tau)$ is anti-correlated with the density fluctuation $\rho(X, \tau)$, and the local maxima of $T$ correspond to the regions where the density is lower. On the other hand, for $K$ larger and close to $K=\pi$, the temperature maxima occur in correspondence with the density maxima, thus we observe that the relaxation time of the inelastic system is shorter than the relaxation time for $\alpha=1$. 


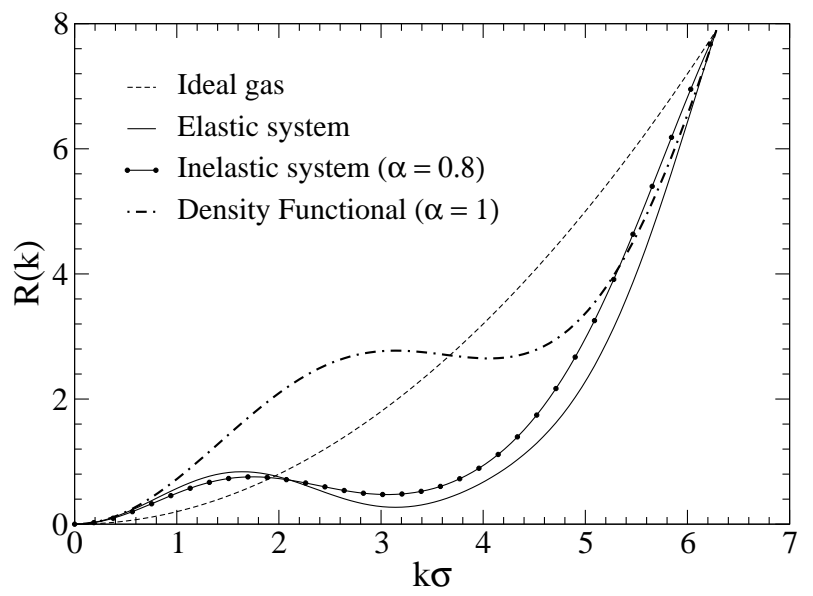

FIG. 2: Decay rate, $R(K)$ of a small sinusoidal density perturbation around a constant background $\rho_{0}=0.68$ as a function of the the reduced wave-vector $K$. The dimensionless friction constant is $\Gamma=5$. The non monotonic dependence of $R(K)$ on $K$ increases as the density increases. Therefore collisions can accelerate or slow down the relaxation with respect to an ideal gas behavior. The dashed line represents the decay rate of a system of non interacting particles, the dash-dot line the Density functional result, the full line the system with $\alpha=1$, and the dot-line the inelastic system with $\alpha=0.8$.

\section{B. Numerical tests}

To validate the theory we shall compare the predictions of Eq. (25) with those obtained by a numerical solution of the dynamical equations for the trajectories of the particles, using the algorithm illustrated in Ref. 22. The comparison is performed by considering an ensemble of "noise" histories (typically $10^{4}$ ) and averaging the observables over such an ensemble. The first category of checks concerns the homogeneous static properties of the system, namely temperature and pressure. The dependence of the temperature on the density predicted by Eq. (41) is shown in Fig. 33, where it is displayed against the numerical results obtained at two different values of the coefficient of restitution. A similar comparison between pressure [Eq. (40)] and the simulation results are reported in Fig. 4, showing a satisfactory agreement. The second category concerns the inhomogeneous static properties, which can be probed by measuring

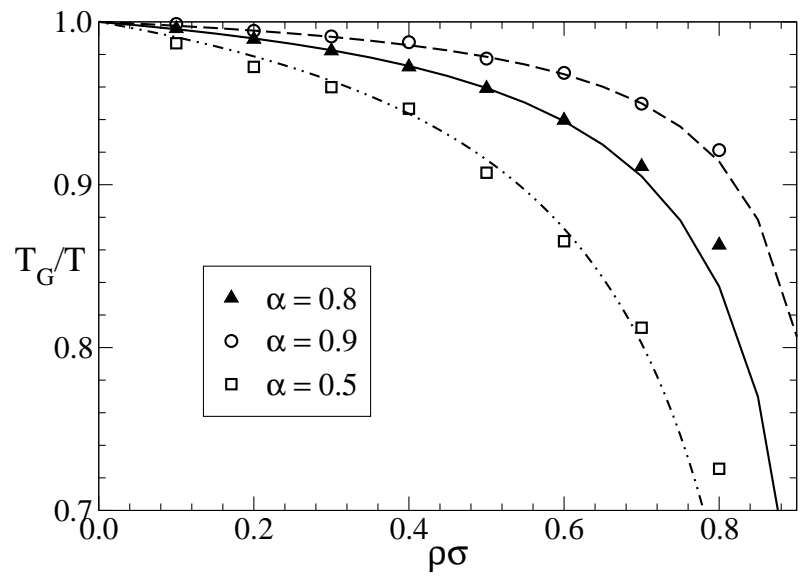

FIG. 3: Uniform system: temperature versus reduced density for $\alpha=0.9, \alpha=0.8$ and $\Gamma=5$. Comparison between Brownian dynamics simulations (points) and the predictions of our theory (lines).

the response of the model to some specific non-uniform external perturbations.

The third category of tests aims, instead, to probe some genuinely time-dependent properties of the system and we have chosen as examples the free expansion of a packet of particles initially localized in a narrow region and the escape of a packet from a potential well. 


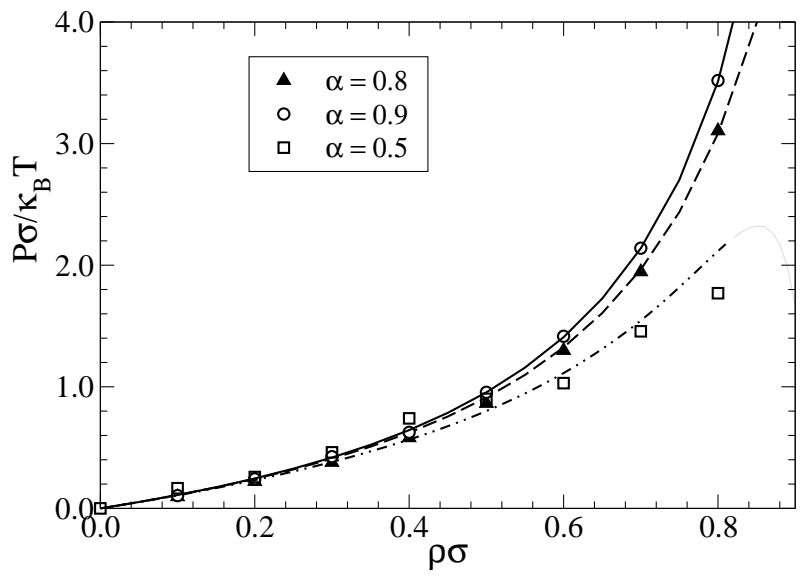

FIG. 4: Uniform system: pressure versus reduced density for $\alpha=0.9, \alpha=0.9$ and $\Gamma=5$. Comparison between Brownian dynamics simulations (points) and the predictions of our theory (lines).

\section{Inhomogeneous Steady State Properties}

Soft repulsive potential. We consider, first, a fixed external potential of the form

$$
V(X)=V_{0} \tanh (X / \xi)
$$

representing a soft repulsive wall located at $X=0$ and characterized by a softness parameter $\xi=0.2$ and height $V_{0}=4$. In Fig. 5 we compare the density profiles, obtained from the stationary solution of the dynamical Eq. (25), for two values of coefficient of restitution and for $\Gamma=5$, with the corresponding profiles extracted from molecular dynamics simulation. The wall perturbs the fluid by inducing a non monotonic profile and a stationary state is achieved when hydrostatic equilibrium is reached.

Both the MD and the integral equation reveal the same feature: near the wall the elastic density profile is slightly higher than the corresponding profile with $\alpha=0.8$. Intuitively such a difference can be understood by considering that the bulk pressures, approximately proportional to the corresponding values of the the density near the wall, display a similar difference. The temperature profile, shown in Fig. 66, varies non monotonically from the value inside the wall to a lower value in the bulk and is the signature of the non equilibrium nature of the system.

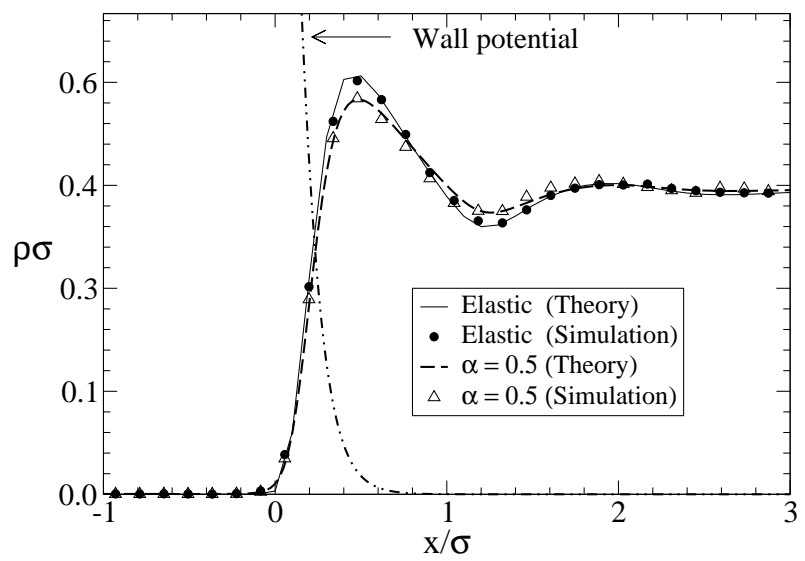

FIG. 5: Density profiles in the presence of a repulsive soft wall at $x=0$, indicated by a dashed line, and implemented in the simulations through the potential $V(X)=V_{0} \tanh (X / \xi)$ with $V_{0}=4$. Data refer to $\alpha=1, \alpha=0.5$ and $\Gamma=5$. Points indicate the results of the simulations whilst the lines are the corresponding results from our theory. The agreement between simulation an theory is excellent and it is also interesting to note that the elastic system $(\alpha=1)$ presents a higher peak near the wall corresponding to a larger pressure exerted, toward the wall, on each particle by the rest of the system.

We study, now, the stationary profile induced by a static periodic external potential of the form

$$
V(X)=V_{0} \cos \left(\frac{2 \pi}{w} X\right)
$$




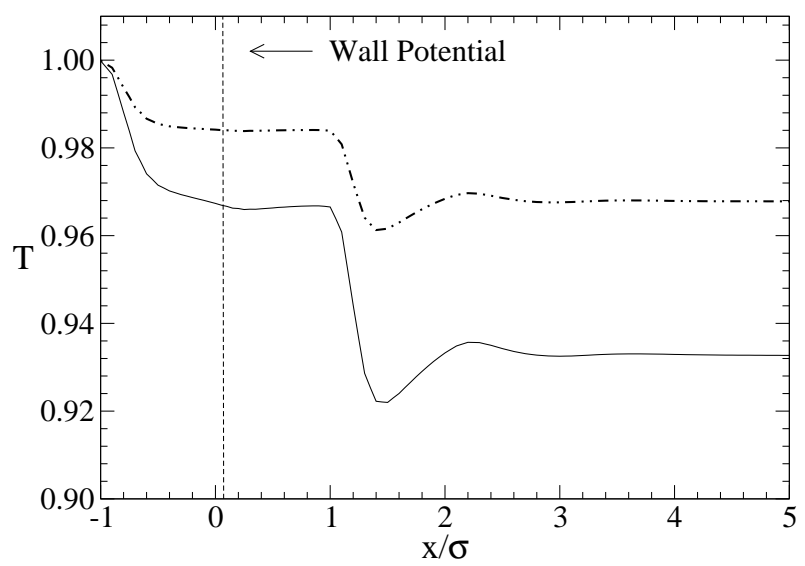

FIG. 6: Temperature profiles in the presence of the same repulsive soft wall of Fig. 5 for a system with $\Gamma=5$ and inelasticity $\alpha=0.8, \alpha=0.5$.

For moderate values of the bulk packing fraction the asymptotic value of the induced density profile turns out to be modulated with the same period as the potential. The height of the peaks is lower than the corresponding height of the non-interacting case, because the hard-core repulsion tends to smear the particles over the wells away from the minimum energy configuration. On the other hand, one can appreciate a difference between the elastic and the inelastic case. The latter displays peaks slightly higher and narrower as shown in Fig. 7, a fingerprint of the tendency toward clustering induced by the inelasticity of collisions.

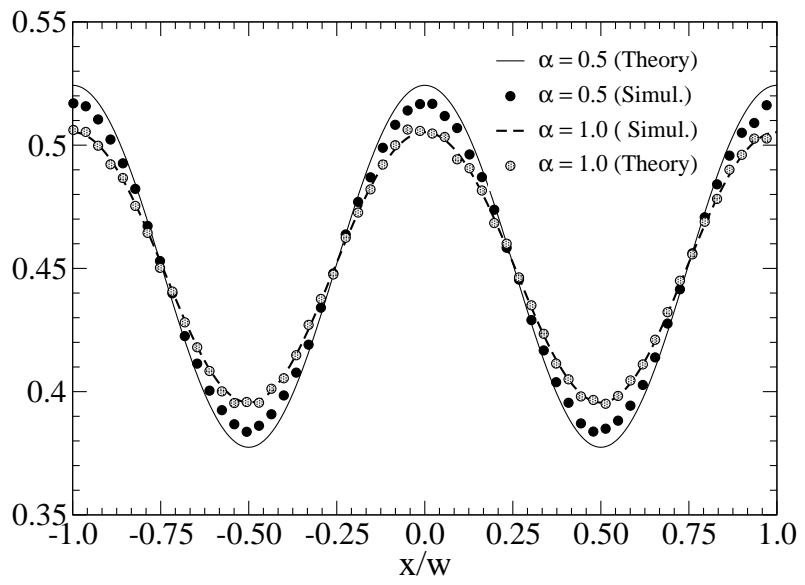

FIG. 7: Density profiles of a system with average density $\rho=0.45$ in an external potential defined by Eq. (46) with $w=8$ and $\Gamma=5$. Black circles correspond to simulation results with $\alpha=0.8$, while shaded circles refer to simulations with $\alpha=1.0$. Dashed and full lines indicate the corresponding results from the present theory for $\alpha=1.0$ and $\alpha=0.8$ respectively.

\section{Inhomogeneous Dynamical Properties}

We consider the free expansion of $N$ hard-rods in the absence of external fields. In Fig. 8, we display the evolution of the variance of the positions of the particles, with respect to their center of mass, $W=\frac{1}{N} \sum_{i}\left\langle\left(x_{i}-x_{c m}\right)^{2}\right\rangle$ for different values of the inelasticity $\alpha$. The average is meant over different and independent noise realizations. In the case of free particles $D=\Gamma^{-1}$, we observe a linear growth of this quantity, which is well described by the diffusive law, $W(\tau)=2 D \tau$. In agreement with our analytical prediction of Sec. VI, the coefficient $D$ is a growing function of the coefficient of restitution and of the number of particles. The first effect can be interpreted by noticing that smaller values of $\alpha$ correspond to larger dissipation and thus to lower local kinetic temperatures. Since one expects $D$ to be proportional to the ratio between temperature and friction coefficient a lowering of the kinetic temperature determines a decrease of the spreading. On the other hand, one can compare the spreading of the same initial configuration in 
the case of non-interacting particles. Figure 8 shows that the ideal gas case corresponds to a diffusion slower of all cases where the hard core repulsion is at work. Finally, the importance of the corrections to the DDF equation, can be appreciated by rescaling the data corresponding to different values of $\Gamma$ according to the formula $W / \Gamma$. The free particle case, of course gives a perfect collapse, whereas the interacting cases display increasing deviations as $\Gamma$ decreases.

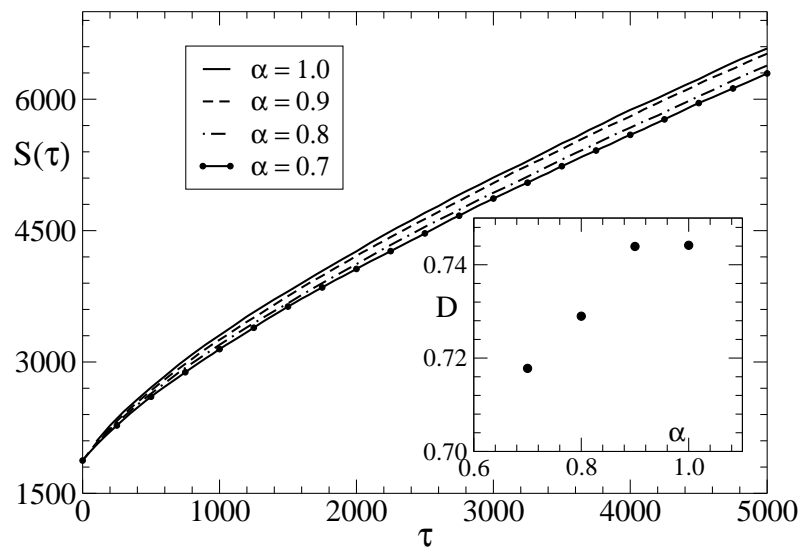

FIG. 8: Free expansion of a cluster of 128 particles initially concentrated over a region of size $150 \sigma$. The curves represent the time growth of the variance of the particle distribution with respect to their center of mass for inelastic systems with different values of $\alpha$ but same $\Gamma=5$. The inset shows the values of the diffusion coefficient estimated by the asymptotic slope of the curves.

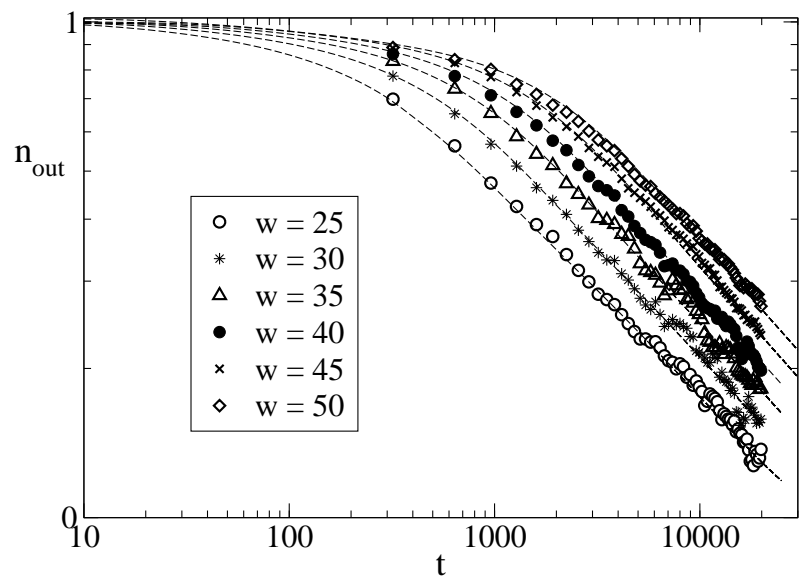

FIG. 9: Time decay of the number of particles initially located in a single well of width $w$ for $\alpha=0.8, \Gamma=5$. The same numerical experiment is repeated for different well sizes but maintaining fixed the barrier height. Broken lines represent the theoretical predictions and the symbols the simulations results obtained as an average over 300 independent runs.

Finally, we consider the escape process of a group of particles from a well of the sinusoidal potential. This numerical experiment amounts to preparing initially a set of particles in a potential well and measuring the fraction $n_{\text {in }}(t)$ that populates the well at that instant. Figure 9 reports the log-log plot of decay in time of $n_{\text {in }}(t)$, obtained by averaging over 300 independent runs, for different well widths $w$. For comparison we also show the corresponding quantity computed through the DDFT (dashed lines) which correctly reproduces the dynamical features of the escape process. We see that the rate at which particles exit the original well decreases with $w$, because the particles spend more time in that well. The collisionless particle systems generally displays a longer escape time than interacting systems, because the absence of excluded volume effects does not entail an effective reduction of the depth of the potential well, which, instead, is the relevant feature characterizing the escape experiments involving hard core particles. The inelasticity, on the other hand, tends to decrease the escape rate due to the energy loss caused by collisions, but this does not counterbalance the excluded volume effect so that the rate of the inelastic particles remains faster than the corresponding rate of the collisionless model. 


\section{CONCLUSIONS}

In this paper we have derived a method to study the dynamics of an assembly of particles interacting inelastically, and driven by a stochastic thermostat. We have found that the particles adopt spatial configurations which are very close to those of an equilibrium system, in spite of the fact that our system is driven and dissipative. The reason for such a similarity is twofold as suggested by a recent study of Reis et al $\underline{\underline{44}}$ : the homogeneous energy feeding mechanism and the importance of the repulsive forces. However, the present work shows that there is no need to invoke entropic forces to explain the observed inhomogeneities. A kinetic approach, in which the short range repulsion is suitably accounted for by means of a suitable treatment of spatial correlations, predicts fairly well the observed structural properties 45 . Our theory indicates that the steady state configurations occur not as a result of the minimization of some hypothetical coarse grained free energy functional but as a result of the competition between the uniform energy injection and the energy dissipation. These two effects are described by an Enskog collision operator and by a a Fokker-Planck collision operator, respectively. The evolution of the phase space distribution function is thus governed by a Fokker-Planck-Enskog (FPE) equation, whose solution still remains an extremely difficult task for dense fluids due to the complexity of the collision kernel and to the computer resources needed to resolve the distribution function. However, when the friction is sufficiently high one can derive a simpler description by an iterative elimination of the fast degrees of freedom, such as the velocities of the particles. Such a procedure is based on the intuition that these achieve locally their equilibrium distribution, whereas the positions evolve more slowly. Truncating this iteration at the first order in the inverse friction parameter $\Gamma^{-1}$ is equivalent to approximating the velocity distribution functions by Maxwellians at temperatures equal to that of the heat bath. Further terms, associated with non Maxwellian contributions to the velocity distribution function, are included in the expansion and contribute to the evolution. The result is a self-consistent time dependent equation for the local density, where the "internal field" is determined by the density itself either through the standard hard-rod entropic contribution or by the velocity and energy currents generated by spatial density gradients.

At a technical level Eq. (25) is derived by applying a multiple time-scale method to the Fokker-Planck-Enskog equation. The resulting equation bears a strong similarity with the Dynamic Density Functional equation, but it is not based on the notion of coarse grained Free Energy, a concept which cannot be applied to open non equilibrium systems, such as the inelastic fluid we have studied in this work. Equation (25) is a density functional equation for the density field $\phi_{00}(X, \tau)$ where its evolution depends on a functional of $\phi_{00}$ itself and its derivatives. It is local in time, but does not possess a generating Liapunov functional, therefore, we cannot prove that the associated dynamics minimizes some cost function.

In more detail we have found that:

a) the inelasticity induces changes even in the stationary properties of the fluid with respect to the elastic reference system to order $\Gamma^{-1}$. The changes can be observed both in the velocity distribution and in the structure of the non uniform fluid.

b) Whereas in the description of a colloidal fluid the friction $\Gamma$ is relevant only for the relaxation properties, in the inelastic fluid $\Gamma$ determines its stationary properties also.

c) The theory holds in the region $\omega_{E} / \gamma<1$, when the typical time-scale of the heat bath is shorter than the Enskog collision frequency.

As far as future perspectives are concerned the method can be generalized to higher dimensions and different types of inter-particle forces and to systems with a non uniform distribution of heat sources 46 . A second type of generalization consists in performing the same multiscale expansion at the level of the two particle phase-space distribution function, by truncating the BBGKY hierarchy one step further, which would allow us to compute self-consistently the pair correlation function of the system.

\section{ACKNOWLEDGMENTS}

UMBM acknowledges a grant COFIN-MIUR 2005, 2005027808. PT acknowledges grants FIS2004-05035-C03-02 by the Direccion General de Investigacion of Spain, and S-0505/ESP/0299 by the Comunidad Autonoma de Madrid.

\section{APPENDIX A: COLLISION INTEGRALS}

In this appendix we show how to perform the velocity integrations and reduce the collision integrals to simple functions of space and time only. Using the definition of collision integral given in the text by Eq. (8) and setting 
$u=V_{2}-V$, we obtain the following explicit expression:

$$
\begin{aligned}
C_{n}(X, \tau) & =g_{2}(X, X+1)\left\{\int _ { - \infty } ^ { \infty } d V \mu _ { n } ( V ) \left[\int_{-\infty}^{0} d u u P(X, V, \tau) P(X+1, u+V, \tau)\right.\right. \\
& \left.\left.+\frac{1}{\alpha^{2}} \int_{0}^{\infty} d u u P(X, V+s u, \tau) P(X+1, V+q u, \tau)\right]\right\} \\
& -g_{2}(X, X-1)\left\{\int _ { - \infty } ^ { \infty } d V \mu _ { n } ( V ) \frac { 1 } { \alpha ^ { 2 } } \left[\int_{-\infty}^{0} d u u P(X, V+s u, \tau) P(X-1, V+q u, \tau)\right.\right. \\
& \left.\left.+\int_{0}^{\infty} d u u P(X, V, \tau) P(X-1, u+V, \tau)\right]\right\},
\end{aligned}
$$

where $s=(1+\alpha) / 2 \alpha, q=-(1-\alpha) / 2 \alpha, \mu_{0}(V)=1, \mu_{1}(V)=V$ and $\mu_{2}(V)=V^{2} / 2$. After substituting the expression of $P(X, V, \tau)$ in terms of its partial amplitudes into Eq. (A1), one can eliminate the velocities obtaining:

$$
\begin{aligned}
C_{n}(X, \tau) & =g_{2}(X, X+1) \sum_{\mu, \nu}\left[N_{\mu \nu}^{(n)}(\alpha=1)+\frac{1}{\alpha^{2}} M_{\nu \mu}^{(n)}(\alpha)\right] \Phi_{\mu}(X, \tau) \Phi_{\nu}(X+1, \tau) \\
& -g_{2}(X, X-1) \sum_{\mu, \nu}\left[M_{\mu \nu}^{(n)}(\alpha=1)+\frac{1}{\alpha^{2}} N_{\nu \mu}^{(n)}(\alpha)\right] \Phi_{\mu}(X, \tau) \Phi_{\nu}(X-1, \tau) .
\end{aligned}
$$

where the matrix elements $M_{\mu \nu}^{(n)}$ and $N_{\mu \nu}^{(n)}$ are defined as:

$$
\begin{aligned}
& N_{\mu \nu}^{(n)}(\alpha)=\int_{-\infty}^{0} d u u \int_{-\infty}^{\infty} d V \mu_{n}(V) H_{\mu}(V+q u) H_{\nu}(V+s u) \\
& M_{\mu \nu}^{(n)}(\alpha)=\int_{0}^{\infty} d u u \int_{-\infty}^{\infty} d V \mu_{n}(V) H_{\mu}(V+q u) H_{\nu}(V+s u) .
\end{aligned}
$$

and have the symmetry property

$$
N_{\mu \nu}^{(n)}(\alpha)=(-1)^{\mu+\nu+n+1} M_{\mu \nu}^{(n)}(\alpha)
$$

so that it is sufficient to calculate only the matrix elements of $M_{\mu \nu}^{(n)}(\alpha)$ in order to compute Eq. (A2):

$$
\begin{gathered}
\frac{M_{\mu \nu}^{(1)}(\alpha)}{\alpha^{2}}=\left|\begin{array}{ccc}
-\alpha / 2 & \frac{1-2 \alpha}{2 \sqrt{\pi}} & \frac{1}{2}(1-\alpha) \\
\frac{1}{2 \sqrt{\pi}}(1+2 \alpha) & \alpha / 2 & -\frac{1}{4 \sqrt{\pi}}(1-2 \alpha) \\
-\frac{1}{2}(1+\alpha) & -\frac{1}{4 \sqrt{\pi}}(1+2 \alpha) & 0
\end{array}\right| . \\
\frac{M_{\mu \nu}^{(2)}(\alpha)}{\alpha^{2}}=\left|\begin{array}{ccc}
-\frac{1}{4 \sqrt{\pi}}\left(1-2 \alpha^{2}\right) & -\frac{1}{8}\left(1+2 \alpha-3 \alpha^{2}\right) & \frac{1}{8 \sqrt{\pi}}\left(1-8 \alpha+6 \alpha^{2}\right) \\
\frac{1}{8}\left(1-2 \alpha-3 \alpha^{2}\right) & \frac{3}{8 \sqrt{\pi}}\left(1-2 \alpha^{2}\right) & \frac{1}{8}(1-\alpha)(1+3 \alpha) \\
\frac{1}{8 \sqrt{\pi}}\left(1+8 \alpha+6 \alpha^{2}\right) & -\frac{1}{8}(1+\alpha)(1-3 \alpha) & -\frac{3}{16 \sqrt{\pi}}\left(1-2 \alpha^{2}\right)
\end{array}\right| .
\end{gathered}
$$

In addition, we verify that in the case $n=0$ the following combinations vanish:

$$
\begin{aligned}
& N_{\mu \nu}^{(0)}(\alpha=1)+\frac{1}{\alpha^{2}} M_{\nu, \mu}^{(0)}(\alpha)=0 \\
& M_{\mu \nu}^{(0)}(\alpha=1)+\frac{1}{\alpha^{2}} N_{\nu \mu}^{(0)}(\alpha)=0
\end{aligned}
$$

so that $C_{0}(X, \tau)=0$, since collisions conserve the number of particles. 


\section{APPENDIX B: MULTIPLE TIME SCALE METHOD.}

Our previous work ${ }^{11}$ has extended to the case of colliding particles a method to derive the Smoluchowski equation starting from the Kramers equation. It was originally proposed in the 1970s for a gas of non-interacting particles by Titulaer $\underline{47}$ and nicely reviewed by Bocquet $\underline{48,49}$.

It represents a particular application of multiple time-scale analysis 50 designed to handle singular perturbations. In the present case the singularity stems from the fact that when $\Gamma>>1$ the time derivative occurs among the small terms of Eq. (14).

Because the inelasticity brings about some remarkable new features we shall report the derivation of the salient parts of the multiple scale method in this particular case. The multiple time-scale analysis introduces a set of auxiliary time scales $\tau_{n}=\Gamma^{-n} \tau$, with $n=0,1,2, \ldots$. The $\tau_{n}$ are treated as independent variables so that the time derivative with respect to $\tau$ is replaced by

$$
\frac{\partial}{\partial \tau}=\frac{\partial}{\partial \tau_{0}}+\frac{1}{\Gamma} \frac{\partial}{\partial \tau_{1}}+\frac{1}{\Gamma^{2}} \frac{\partial}{\partial \tau_{2}}+
$$

The partial amplitudes $\Phi_{\nu}(X, \tau)$ and the collision terms $C_{\nu}(X, \tau)$ are also treated as functions of the auxiliary time scales and expanded perturbatively as:

$$
\Phi_{\nu}\left(X, \tau_{0}, \tau_{1}, \tau_{2}, . .\right)=\sum_{n=0}^{\infty} \frac{1}{\Gamma^{n}} \phi_{n \nu}\left(X, \tau_{0}, \tau_{1}, \tau_{2}, . .\right)
$$

and

$$
C_{\nu}\left(X, \tau_{0}, \tau_{1}, \tau_{2}, . .\right)=\sum_{n=0}^{\infty} \frac{1}{\Gamma^{n}} c_{n \nu}\left(X, \tau_{0}, \tau_{1}, \tau_{2}, . .\right)
$$

By substituting Eqs. (B1)-(B3) into Eq. (14) and equating equal powers of $\Gamma$, one obtains iteratively a series of equations which must be satisfied by the coefficients $\phi_{s \nu}\left(X, \tau_{0}, \tau_{1}, \tau_{2}, ..\right)$ and $c_{s \nu}\left(X, \tau_{0}, \tau_{1}, \tau_{2}, ..\right)$. The latter coefficients are obtained using the formula:

$$
\begin{gathered}
c_{s \nu}(X, \tau)=\sum_{l+m=s} \sum_{\mu, \nu} g_{2}(X, X+1)\left[N_{\mu \nu}^{(n)}(\alpha=1)+\frac{1}{\alpha^{2}} M_{\nu \mu}^{(n)}(\alpha)\right] \phi_{l \mu}(X, \tau) \phi_{m \nu}(X+1, \tau) \\
-g_{2}(X, X-1)\left[M_{\mu, \nu}^{(n)}(\alpha=1)+\frac{1}{\alpha^{2}} N_{\nu \mu}^{(n)}(\alpha)\right] \phi_{l \mu}(X, \tau) \phi_{m \nu}(X-1, \tau) .
\end{gathered}
$$

Notice that the $c_{s \nu}$ 's are functionals of the $\phi_{s \nu}$ 's.

We begin with the order $\Gamma^{0}$ :

$$
L_{F P}\left[\sum_{\nu} \phi_{0 \nu} H_{\nu}\right]=0
$$

having the solution $\phi_{0 \nu}=0$ for $\nu \neq 0$, which inserted in Eq. (9) determines the expansion coefficients of order $\Gamma^{-1}$ in terms of $\phi_{0 \nu}=0$.

$$
\begin{aligned}
L_{F P}[ & \left.\phi_{11} H_{1}+\phi_{12} H_{2}+\phi_{13} H_{3}+\phi_{14} H_{4}+. .\right]= \\
& \frac{\partial \phi_{00}}{\partial \tau_{0}} H_{0}+D_{X} \phi_{00} H_{1}-c_{01} H_{1}-c_{02} H_{2}-c_{03} H_{3}-c_{04} H_{4} . \cdot
\end{aligned}
$$

where we have employed the abbreviation $D_{X} \equiv\left(\partial_{X}-F(X)\right)$. We also perform our expansion by setting $\phi_{s 0}=0$ for all $s>0$. By equating the coefficients of the same $H_{\nu}$ in Eq. (B7) we find the following relations

$$
\begin{gathered}
\frac{\partial \phi_{00}}{\partial \tau_{0}}=0 \\
\phi_{11}=-D_{X} \phi_{00}+c_{01}
\end{gathered}
$$


and for $\nu>1$

$$
\phi_{1 \nu}=\frac{1}{\nu} c_{0 \nu} .
$$

The procedure can be iterated to the order $\Gamma^{-2}$, writing the equation

$$
\begin{aligned}
& L_{F P}\left[\sum_{\nu \geq 1} \phi_{2 \nu} H_{\nu}\right]=\sum_{\nu \geq 1} \frac{\partial \phi_{1 \nu}}{\partial \tau_{0}} H_{\nu}+\frac{\partial \phi_{00}}{\partial \tau_{1}} H_{0}+D_{X} \phi_{11} H_{2}+\partial_{X} \phi_{11} H_{0}+ \\
& D_{X} \phi_{12} H_{3}+2 \partial_{X} \phi_{12} H_{1}+D_{X} \phi_{13} H_{4}+3 \partial_{X} \phi_{13} H_{2}+D_{X} \phi_{14} H_{5}+4 \partial_{X} \phi_{14} H_{3}-\sum_{\nu \geq 1} c_{1 \nu} H_{\nu}
\end{aligned}
$$

which leads to the following conditions

$$
\begin{gathered}
\frac{\partial \phi_{00}}{\partial \tau_{1}}=-\partial_{X} \phi_{11} \\
\frac{\partial \phi_{11}}{\partial \tau_{0}}=-\phi_{21}-2 \partial_{X} \phi_{12}+c_{11}=0 \\
\frac{\partial \phi_{12}}{\partial \tau_{0}}=-2 \phi_{22}-D_{X} \phi_{11}-3 \partial_{X} \phi_{13}+c_{12}=0
\end{gathered}
$$

and

$$
\frac{\partial \phi_{13}}{\partial \tau_{0}}=-3 \phi_{23}-D_{X} \phi_{12}-4 \partial_{X} \phi_{14}+c_{13}=0 .
$$

The amplitude $\phi_{11}$, being a functional of $\phi_{00}$, does not depend on $\tau_{0}$. Hence, the 1.h.s. of Eq. (B12) vanishes and we find

$$
\phi_{21}=-2 \partial_{X} \phi_{12}+c_{11} .
$$

Similarly, we obtain for $\nu>1$ :

$$
\phi_{2 \nu}=-\frac{1}{\nu}\left[D_{X} \phi_{1(\nu-1)}+(\nu+1) \partial_{X} \phi_{1(\nu+1)}+c_{1 \nu}\right]
$$

Explicitly we write

$$
\begin{gathered}
\phi_{22}=-\frac{1}{2}\left[D_{X} D_{X} \phi_{00}+D_{X} c_{01}+\partial_{X} c_{03}-c_{12}\right] \\
\phi_{23}=-\frac{1}{3}\left[\frac{1}{2} D_{X} c_{02}+\partial_{X} c_{04}-c_{13}\right] \\
\phi_{24}=-\frac{1}{4}\left[\frac{1}{3} D_{X} c_{03}+\partial_{X} c_{05}-c_{14}\right] .
\end{gathered}
$$

Finally, by equating the coefficients of $H_{0}$, we arrive to the equation:

$$
\frac{\partial \phi_{00}}{\partial \tau_{1}}=\partial_{X}\left[D_{X} \phi_{00}-c_{01}\right] .
$$

In order to carry out the derivative of $\phi_{00}$ with respect to the time $\tau_{2}$, we iterate the procedure to the order $\Gamma^{-3}$ by writing:

$$
\begin{gathered}
L_{F P}\left[\sum_{\nu \geq 1} \phi_{3 \nu} H_{\nu}\right]=\frac{\partial}{\partial \tau_{0}} \sum_{\nu \geq 1} \phi_{2 \nu} H_{\nu}+\frac{\partial}{\partial \tau_{1}} \sum_{\nu \geq 1} \phi_{1 \nu} H_{\nu}+\frac{\partial \phi_{00}}{\partial \tau_{2}} H_{0} \\
+\sum_{\nu \geq 1}\left[D_{X} \phi_{2 \nu} H_{\nu+1}+\nu \partial_{X} \phi_{2 \nu} H_{\nu-1}\right]-\sum_{\nu \geq 1} c_{2 \nu}
\end{gathered}
$$


and equating the coefficients of $H_{0}(V)$ we obtain the following equation

$$
\frac{\partial \phi_{00}}{\partial \tau_{2}}=-\partial_{X} \phi_{21}=-\partial_{X} c_{11}+\partial_{X}^{2} c_{02}
$$

where the second equality follows from eqs.(B9) and (B15).

We, now, collect together the different orders in $\tau_{n}$, given by Eqs. (B1), (B11) and (B22), thus restoring the original physical time $\tau$ to find the evolution equation for the density amplitude (25).

${ }^{1}$ H.M. Jaeger, S.R. Nagel, and R.P. Behringer, Rev. Mod. Phys. 68, 1259 (1996).

${ }^{2}$ H.M. Jaeger, S.R. Nagel, and R.P. Behringer, Phys. Today 49(32), 3 (1996).

${ }^{3}$ L.P. Kadanoff, Rev. Mod. Phys. 71, 435 (1999).

4 J. Duran, Sands, Powders and Grains: An Introduction to the Physics of Granular Materials (Springer, New York, 2000).

5 Granular Gases, Lecture Notes in Physics vol 564, edited by T. Poechel and S. Luding (Springer, Berlin, 2001).

6 A. Kudrolli, Rep. Progr. Phys. 67, 209 (2004).

7 D. Paolotti, C. Cattuto, U. Marini-Bettolo-Marconi and A. Puglisi, Granular Matter 5, 75 (2003).

8 S.A. Hill and G.F. Mazenko, Phys. Rev. E 67, 061302 (2003).

9 R. Evans. Adv. Phys. 28, 143 (1979).

10 U. Marini-Bettolo-Marconi and P. Tarazona, J. Chem. Phys. 124, 164901 (2006).

11 U. Marini-Bettolo-Marconi and P. Tarazona, J. Chem. Phys. 110, 8032 (1999), and J. Phys.: Condens. Matter 12, 413 (2000).

12 H. van Beijeren and M.H. Ernst, Physica A, 68, 437 (1973), 70, 225 (1973).

13 T.P.C. van Noije and M.H. Ernst, in Granular Gases edited by T. Poeschel and S. Luding, (Springer, Berlin, 2001).

14 Y. Du, H. Li, and L.P. Kadanoff, Phys. Rev. Lett. 74, 1268 (1995).

15 N. Sela and I. Goldhirsch, Phys. Fluids 7, 507 (1995).

16 S. McNamara and W.R. Young, Phys. Fluids A 4, 496 (1992); and 5, 34 (1993).

17 D.R.M. Williams and F.C. MacKintosh, Phys. Rev. E 54, R9 (1996).

18 A. Barrat, T. Biben, Z. Racz, E. Trizac, F. van Wijland, J. Phys. A: Math. Gen. 35, 463 (2002).

19 E. Ben-Naim, S.Y. Chen, G.D. Doolen, and S. Redner Phys. Rev. Lett. 83, 4069-4072 (1999).

20 A. Baldassarri, U. Marini-Bettolo-Marconi, and A. Puglisi, Europhys. Lett. 58, 14 (2002).

21 A. Santos and M.H. Ernst, Phys. Rev. E 68, 011305 (2003).

${ }^{22}$ F. Cecconi, F. Diotallevi, U. Marini-Bettolo-Marconi, and A. Puglisi, J. Chem. Phys. 120, 35 (2004); 121, 5125 (2004).

${ }^{23}$ U. Marini-Bettolo-Marconi, M. Natali, G. Costantini and F. Cecconi, J. Chem. Phys. 124, 044507, (2006).

${ }^{24}$ G. Costantini, F. Cecconi, and U. Marini-Bettolo-Marconi, J. Chem. Phys. 125, 204711 (2006).

25 J.M. Montanero and A. Santos, Granular Matter 1, 57, (1998).

26 A. Santos, Physica A 321, 442 (2003).

27 A. Pagnani, U. Marini-Bettolo-Marconi and A. Puglisi, Phys. Rev. E 66, 051304 (2002).

28 H. Hayakawa, Phys. Rev. E 68, 031304 (2003).

${ }^{29}$ F. Cecconi, A. Puglisi, U. Marini-Bettolo-Marconi and A. Vulpiani, Phys. Rev. Lett. 90064301 (2003).

30 J.A. Carrillo, C. Cercignani, and I.M. Gamba, Phys. Rev. E 62, 7700 (2000).

31 A. Santos, J.M. Montanero, J.W. Dufty and J.J. Brey, Phys. Rev. E 57, 1644 (1998), and J.W. Dufty, A. Santos, and J. Brey, Phys. Rev. Lett. 77, 1270 (1996).

32 V. Garzó and J.W. Dufty, Phys. Rev. E 59, 5895 (1999).

33 J.K. Percus, J. Stat. Phys. 15, 505 (1976).

34 We remark that in the present model two features concur to remove the pathological aspects of one dimensional hydrodynamics: the stochastic driving and the inelasticity of collisions.

35 D. Benedetto, E. Caglioti, and M. Pulvirenti, Math. Modell. Numer. Anal.31, 615 (1997).

36 E.L. Grossman, T. Zhou and E. Ben-Naim, Phys. Rev. E 554200 (1997).

37 H.Risken, The Fokker-Planck equation (Springer-Verlag, Berlin, 1984).

38 C. Gardiner, Handbook of stochastic methods for physics, chemistry and in the natural sciences (Springer-Verlag, Berlin, 1994).

39 N. van Kampen, Stochastic Processes in Physics and Chemistry, (North-Holland, Amsterdam, 1992).

40 H.A. Kramers, Physica A, 7, 284 (1940).

41 M. von Smoluchowski, Ann. Phys., 48, 1103 (1916).

42 G. Wilemski, J. Stat. Phys., 14, 153 (1976).

43 D. Enskog, Kungl. Sv. Vetenskaosakad. Handl. 63, No. 4 (1922).

44 P.M. Reis, R.A. Ingale, and M.D. Shattuck Phys. Rev. Lett. 96, 258001 (2006).

45 J.S. Olafsen and J.S. Urbach, Phys. Rev. Lett. 95, 098002 (2005).

46 C. López and U. Marini-Bettolo-Marconi Phys. Rev. E 75, 021101 (2007).

47 U.M. Titulaer, Physica A, 91, 321 (1978) and Physica A100, 234 (1980) .

48 L. Bocquet, Am. J. Phys., 65, 140 (1997). 
49 J. Piasecki, L. Bocquet and J.P. Hansen, Physica A, 218, 125 (1995).

50 C.M. Bender and S.A. Orszag, Advanced Mathematical Methods for Scientists and Engineers, (McGraw-Hill, New York, 1978). 